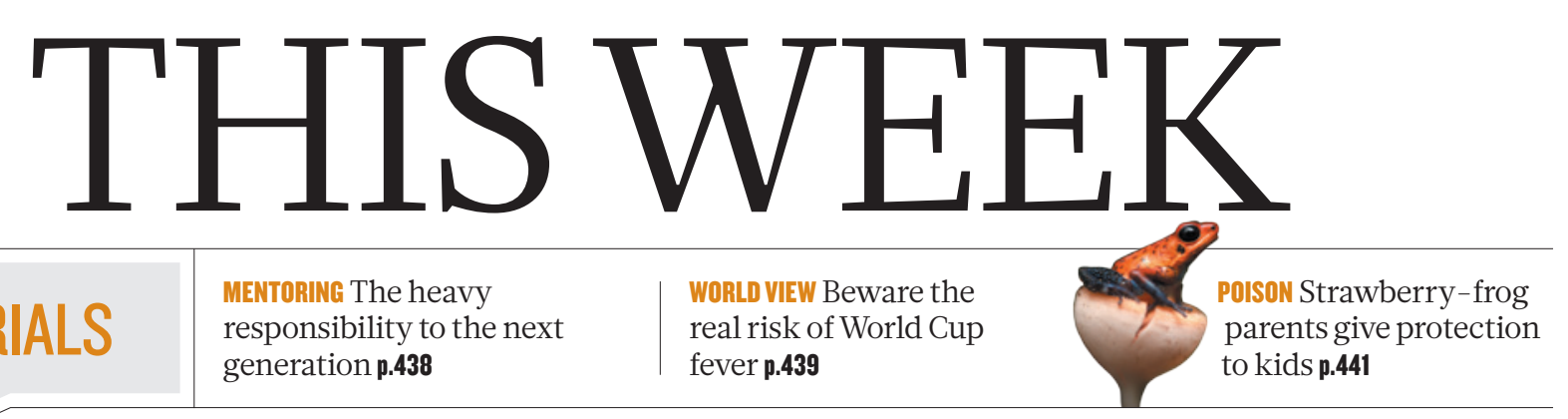

\title{
Nailing fingerprints in the stars
}

\author{
Laboratory-based experiments are sorely needed to complement the rapidly proliferating spectral \\ data originating from observations by the latest space telescopes.
}

$\mathrm{W}$ hat are stars made of? After astronomers detected a brightyellow, unknown spectral line in sunlight in 1868, they named the new element helium after the Greek Sun god Helios. But it was some 30 years before physicists on Earth managed to detect - and so confirm the discovery of - helium in a laboratory.

It is a pattern that has been repeated many times since: the indirect detection of elements and molecules through spectral signatures in space has come ahead of detailed study on the ground. Lab spectroscopy has long lagged behind telescope observations, but it is striking just how wide the gap has now grown.

A cutting-edge infrared spectrograph, for example, installed in 2011 on the Sloan Digital Sky Survey (SDSS) telescope in Sunspot, New Mexico, records the spectra of 1,800 stars per night, most located in the bulge of the Milky Way galaxy, where dust prevents visible wavelengths of light from reaching Earth. The result is the detection of thousands of unidentified spectral lines - dips or peaks of electromagnetic waves at specific energies, caused by absorption of the light by gas on the way to Earth or emission by gas on stars.

Some physicists are now pointing out the irony that multimilliondollar projects such as the SDSS are producing data that cannot be analysed because of a failure to support much cheaper lab work on the ground. They have a point, and support for lab-based research that can decipher such spectra should be increased. A good rule of thumb is that agencies funding telescope projects that are doing cutting-edge spectroscopy should spend a small fraction, maybe a few per cent, of the money on associated lab spectroscopy.

Lab-based measurements are less glamorous, but big questions about the evolution of galaxies will be solved by understanding small but important details about the physics and chemistry of millions of stars as revealed by spectra. For example, spectra could give clues to whether stars in the Galactic bulge formed there or migrated to it later. Spectra can also shed light on the amount of dark matter near a star, by revealing information about the star's motion, which shifts its spectral lines.

A good example of the benefits of such work comes from a November paper in The Astrophysical Journal by atomic physicists at Imperial College London, the National Institute of Standards and Technology in Gaithersburg, Maryland, and the Astrophysical Institute of the Canary Islands in Tenerife, Spain (M. P. Ruffoni et al. Astrophys. J. 779, 17; 2013). They report 28 probabilities of electron transitions between sets of energy levels for the element iron. These can now be used in combination with spectra to estimate the abundance of iron in stars in the Galactic bulge - a step towards determining their ages and where they formed. None had previously been measured in the laboratory.

Such research is necessary because, to identify and quantify elements in space from spectra, astronomers must know the probability that electrons in the elements' atoms will move between energy levels. For light elements with few electrons, such as hydrogen and helium, the probabilities of transitions can be calculated using the rules of quantum mechanics. But heavier elements have many electrons that can participate in transitions - iron has 26, making the probabilities of possible transitions between levels too complex to calculate accurately. Measuring emissions in the lab is the only alternative. Physicists can use tunable lasers to excite electrons into more levels and measure further transitions. This information can then feed back to the astronomical observations. Extra funds would significantly improve this capacity, giving better access to powerful lasers and detectors.

Even as experimentalists face challenges taking lab spectra, there is an astronomical spectroscopy boom. Aside from the infrared instru-

\section{"Measuring} emissions in the lab is the only alternative." ment taking data on the US\$55-million SDSS, astronomers are planning to build gigantic 30-50-metre telescopes, such as the $€ 1$-billion (US\$1.3-billion) European Extremely Large Telescope, to be based near Cerro Paranal, Chile, which will take hundreds of thousands of stellar spectra. Furthermore, NASA's planned \$8.8-billion James Webb Space Telescope, which like the Sloan instrument uses cuttingedge mercury cadmium telluride infrared detectors, will look at stars and, it is hoped, at the atmospheres of planets outside the Solar System. Although the spectra can be used to estimate the amounts of different elements in the atmospheres of stars or planets, a particular area of interest is in identifying molecules, which also emit characteristic spectral lines when they transition between different states.

Other lab-based experiments might even solve one of the longeststanding questions in astronomy: the origin of the diffuse interstellar bands - dips in the spectra of stars caused by diffuse matter spread between the stars and Earth. They are thought to be due to unstable hydrocarbon radicals, the exact mix of which has yet to be made in the laboratory, and they have puzzled astronomers for almost 100 years. How long do researchers want to wait?

\section{The DIY dilemma}

\section{Misconceptions about do-it-yourself biology mean that opportunities are being missed.}

$\mathrm{T}$ The do-it-yourself-biology movement has an image problem. More commonly called DIYbio, it tends to conjure up pictures of T-shirt-clad misfits marshalling limited scientific skill in their basements as they try to make cool-but-fringe things such as glow-in-the-dark plants. Policy-makers take an opposite view: instead of wayward amateurs, they see twisted experts hellbent on harm, engineering pathogens in their garages to unleash upon the world. A 